\title{
Situación actual y perspectiva de la metrología en el sector salud de la provincia Matanzas
}

Current situation and perspective of metrology in the health sector of Matanzas province

Guillermo Ramos Castro. ${ }^{1}$ Delvis Cárdenas Garabito. ${ }^{2}$ \& Angela Ávila Segovia. ${ }^{3}$

\section{DOI: https://doi.org/10.33262/anatomiadigital.v3i2.1185}

\begin{abstract}
.
Metrology is important in the processes that are developed in medical care, as well as in teaching and research to improve the quality of health services results. The objective of this research is to characterize the metrology activity in the province of Matanzas during the period from January 2013 to September 2016 as part of the quality assurance in health services. For this, a retrospective descriptive study was carried out where all the semiannual and annual reports of the activity carried out by the research department were reviewed and sent to the Ministry of public health and the provincial health department.
\end{abstract}

\section{Resumen.}

La Metrología es importante en los procesos que se desarrollan en la atención médica, así como en la docencia y la investigación para elevar la calidad en los resultados de los servicios de salud. El objetivo de esta investigación es caracterizar la actividad de metrología en la provincia de Matanzas durante el período de enero del 2013 a septiembre del 2016 como parte de la garantía de la calidad en los servicios de salud. Para ello se realizó un estudio descriptivo retrospectivo donde se revisaron todos los informes semestrales y anuales de la actividad que realiza el departamento de

\footnotetext{
${ }^{1}$ Ciencias Médicas Mayabeque, Mayabeque, Cuba, gramos@infomed.sld.cu iD https://orcid.org/0000-00027353-0227

${ }^{2}$ Ciencias Médicas Mayabeque, Mayabeque, Cuba,dcardenas@infomed.sld.cu 0002-7353-0228

https://orcid.org/0000-

${ }^{3}$ Ciencias Médicas Mayabeque, Mayabeque, Cuba, aavila@infomed.sld.cu ID https://orcid.org/0000-0002$7353-0229$
} 
The selection and training of personnel allowed during this period that the execution of the contracting of the verification and calibration service by both primary and secondary care units be $100 \%$. The participation in the courses is not high, due to the stability of the staff in the municipal and hospital health units. The development of the activity allowed a better preparation of professionals and technicians linked to biomedical technology. In addition to complying with the strategy of the Ministry of Public Health in order to ensure the correct use of measuring instruments, the accuracy of the measurements and highlights the importance of metrology in biomedical equipment, and how it impacts on the activities oriented to the reliability of the results. investigaciones y envía al Ministerio de salud pública y la dirección provincial de salud. La selección y capacitación del personal permitió durante este periodo que la ejecución de la contratación del servicio de verificación y calibración por parte de las unidades tanto de atención primaria como secundaria esté al $100 \%$. La participación en los cursos no es elevada, debido a la estabilidad del personal en las unidades de salud municipales y hospitalarias. El desarrollo de la actividad permitió una mejor preparación de los profesionales y técnicos vinculados a la tecnología biomédica. Además de cumplir con la estrategia del Ministerio de Salud Pública a fin de garantizar el uso correcto de los instrumentos de medición, la exactitud de las mediciones y resalta la importancia que tiene la metrología en los equipos biomédicos, y como este impacta en las actividades orientadas a la confiablidad de los resultados.

Palabras claves: Atención médica, calidad,

tecnología biomédica, metrología
Keywords: Medical care, quality, biomedical technology, metrology

\section{Introducción.}

Probablemente la Metrología sea la ciencia más antigua con la que el hombre se ha relacionado, el conocimiento sobre su aplicación es una necesidad fundamental en la práctica de todas las profesiones basadas en las ciencias técnicas y naturales, ya que la medición permite conocer de forma cuantitativa las propiedades físicas y químicas de los objetos y fenómenos naturales (Reyes, et al,2011; Saldarriga,2018).

La metrología definida como tecnología y ciencia de la medición según Almeida Rivas (2010) y LLaque-Lopez (2018) ha impactado a toda la sociedad, tanto en la vida cotidiana, como en los ámbitos: económico, político y social, dado que, varias de las actividades que desarrolla el ser humano, implican mediciones en asuntos tales como la ciencia, la seguridad de las personas, el desarrollo tecnológico, la productividad y la competitividad, el bienestar social, la salud y la calidad de vida (Saldarriga,2018). 
Las mediciones son las principales fuentes de información sobre la eficiencia de los procesos tecnológicos, y son la base sobre la cual se fundamentan todas las transacciones comerciales. Se ha visto que el progreso y desarrollo de las distintas formaciones económico-sociales por las que ha transitado la humanidad han estado ligadas a las mediciones (Reyes, et al,2011; Sánchez, 2018).

Según la norma internacional ISO 9001 (2015) en el acápite 7.1.5.2 Trazabilidad de las mediciones refiere que:

Cuando la trazabilidad de las mediciones es un requisito, o es considerada por la organización como parte esencial para proporcionar confianza en la validez de los resultados de la medición, el equipo de medición debe:

Calibrarse o verificarse, o ambas, a intervalos especificados, o antes de su utilización, contra patrones de medición trazables a patrones de medición internacionales o nacionales; cuando no existan tales patrones, debe conservarse como información documentada la base utilizada para la calibración o la verificación.

El cumplimiento de este requisito implica incrementos de la eficiencia en aquellas partes de la organización en que sea posible identificar las especificaciones exigidas por el cliente puesto que la calidad se relaciona con la satisfacción de las necesidades y expectativas del cliente (Hernández, 2019)

Las entidades prestadoras del servicio de salud, entre sus líneas estratégicas establece la Gestión Tecnológica asociada a los equipos biomédicos como fundamental en sus estándares de calidad, entendiendo que la tecnología biomédica hace parte de las herramientas vitales para el desarrollo óptimo de la medicina, ofreciendo mejores resultados en las actividades de prevención, protección, tratamiento, entre otras, esto teniendo presente el buen estado de esta tecnología, desde su adquisición, pasando por su sostenimiento, hasta finalmente el proceso de baja de los mismos, atravesando por diferentes métodos como el aseguramiento de las mediciones durante la utilización de los equipos (Ramírez, et al, 2017).

La metrología de la tecnología biomédica se define como la ciencia dedicada a asegurar la conformidad con las especificaciones de diseño, necesarios para el funcionamiento correcto y veraz de los equipos e instrumentos de medición biomédicos, lo cual asegura un diagnóstico y tratamiento acertado (Pacheco,2014; Mejías, et al,2017).

Un factor clave en la competitividad de las industrias y en general para el mantenimiento de la calidad de vida de los ciudadanos, es la competencia en materia de medida de las generaciones de profesionales. Es necesario promover una "cultura de medición" que acompañe al avance tecnológico y científico del país. 
Igual que para comunicarnos necesitamos de una cultura, un vocabulario y unas reglas gramaticales, para expresar cuantitativamente los resultados de las medidas se necesita de un conocimiento de la medición, un vocabulario, unos conceptos claros y unas normas homogéneas e internacionalmente aceptadas (Ministerio de Industria, Energía y Turismo,2016).

El papel de la metrología se hace relevante cuando el proceso de medición es vital como apoyo en las actividades orientadas a garantizar la seguridad del paciente en las entidades prestadoras del servicio de salud, dado que en la actualidad estas entidades demandan mejores servicios.

Para lograr esto se tienen que controlar los procesos de medición en cada una de las magnitudes biomédicas que intervienen en los instrumentos para tratamiento y diagnóstico, de ahí la importancia de la aplicación de la Metrología Biomédica la cual estudia las mediciones relacionadas con estas magnitudes, que son generadas por el cuerpo humano y traducidas por los instrumentos biomédicos. Al darle una aplicación oportuna al seguimiento de estas magnitudes biomédicas, se podría entregar al paciente una mejor calidad en el servicio que se verá reflejado en su recuperación y a los profesionales del área de la salud, certeza de que los equipos médicos utilizados están operando bajo los estándares de calidad establecidos por el fabricante.

En la actualidad los servicios de salud se basan en una medida cada vez mayor en las mediciones cuantitativas (Ramírez, 2015).

La metrología en nuestro sector debe garantizar que los equipos empleados durante cualquier proceso asistencial funcionen acorde a los parámetros de calidad establecidos por los fabricantes. Esto ofrece seguridad a los profesionales de nuestro sector en los resultados, tratamiento e intervención de los pacientes.

El objetivo del trabajo es caracterizar la actividad de metrología en la provincia de Matanzas durante el período de enero del 2013 a septiembre del 2016 como parte de la garantía de la calidad en los servicios de salud.

Material y métodos

Se realizó un estudio descriptivo retrospectivo en la provincia de Matanzas. Para la realización de este trabajo se revisaron los informes semestrales y anuales de la actividad que realiza el departamento de investigaciones al Ministerio de Salud Pública y la Dirección Provincial de Salud desde enero 2013 a septiembre de 2016.

Se manejaron variables como: metrólogos capacitados y distribución por municipios, se calculó la distribución de frecuencias de cada una de las variables cualitativas. Con los datos obtenidos se realizaron tablas para mostrar los resultados. 


\section{Resultados y discusión}

A partir del análisis efectuado se pudo apreciar que a pesar de la convocatoria realizada la participación en los cursos no es muy alta. El mayor período es el 2013 con un 52,5\% del total de capacitados. A partir de 2014 se observa una disminución, alcanzando valores entre 12,5 y $20 \%$. Esto se debe fundamentalmente a la estabilidad del personal en las unidades municipales y hospitalarias.

Tabla 1. Total, de metrólogos capacitados según año y nivel de atención. Matanzas 2013-2016

\begin{tabular}{|c|c|c|c|c|c|c|c|c|}
\hline \multirow{2}{*}{ Año } & \multicolumn{2}{|c|}{$\begin{array}{c}\text { Atención } \\
\text { Primaria } \\
\text { Salud }\end{array}$} & $\begin{array}{c}\text { Atención } \\
\text { Secundaria } \\
\text { Salud }\end{array}$ & \multicolumn{2}{c|}{$\begin{array}{c}\text { Otras } \\
\text { entidades }\end{array}$} & \multicolumn{2}{c|}{ Total } \\
\hline & No & \% & No & \% & No & \% & No & $\%$ \\
\hline 2013 & 16 & 50 & 3 & 50 & 2 & 100 & 21 & 52,5 \\
\hline 2014 & 6 & 18,8 & 2 & 33,3 & 0 & 0 & 8 & 20 \\
\hline 2015 & 5 & 15,6 & 0 & 0 & 0 & 0 & 5 & 12,5 \\
\hline 2016 & 5 & 15,6 & 1 & 16,7 & 0 & 0 & 6 & 15 \\
\hline Total & $\mathbf{3 2}$ & $\mathbf{1 0 0}$ & $\mathbf{6}$ & $\mathbf{1 0 0}$ & $\mathbf{2}$ & $\mathbf{1 0 0}$ & $\mathbf{4 0}$ & $\mathbf{1 0 0}$ \\
\hline
\end{tabular}

Fuente: Elaboración propia.

Matanzas, Jovellanos y Colón son los de mayor número de personas capacitadas con 22,5 \% y $15 \%$ respectivamente. (Tabla 2 )

Tabla 2. Total, de metrólogos capacitados según municipio y nivel de atención. Matanzas 2013-2016

\begin{tabular}{|l|c|c|c|c|c|}
\hline \multirow{2}{*}{$\begin{array}{c}\text { Total por } \\
\text { Municipio }\end{array}$} & \multicolumn{2}{|c|}{ Total } & $\begin{array}{c}\text { Atención } \\
\text { Primaria } \\
\text { Salud }\end{array}$ & $\begin{array}{c}\text { Atención } \\
\text { Secundaria } \\
\text { Salud }\end{array}$ & $\begin{array}{c}\text { Otras } \\
\text { entidades }\end{array}$ \\
\cline { 2 - 6 } & No & $\%$ & 3 & 4 & 2 \\
\hline Matanzas & 9 & 22,5 & 1 & 0 & 0 \\
\hline Limonar & 1 & 2,5 & 5 & 1 & 0 \\
\hline Jovellanos & 6 & 15 & 1 & 0 & 0 \\
\hline Perico & 1 & 2,5 & 4 & 1 & 1 \\
\hline Colón & 6 & 15 & 2 & 0 & 0 \\
\hline Arabos & 2 & 5 & 1 & 0 & 0 \\
\hline Calimete & 1 & 2,5 & 2 & 1 & 0 \\
\hline Jagüey & 3 & 7,5 & 1 & 0 & 0 \\
\hline Pedro Betancourt & 1 & 2,5 & 5 & 0 & 0 \\
\hline Cárdenas & 5 & 12,5 & 1 & 0 & 0 \\
\hline Unión de Reyes & 1 & 2,5 & & & 0 \\
\hline
\end{tabular}




\begin{tabular}{|l|c|c|c|c|c|} 
Martí & 2 & 5 & 2 & 0 & 0 \\
\hline Ciénaga de Zapata & 2 & 5 & 2 & 0 & 0 \\
\hline Total & $\mathbf{4 0}$ & $\mathbf{1 0 0}$ & $\mathbf{3 0}$ & $\mathbf{7}$ & $\mathbf{3}$ \\
\hline
\end{tabular}

Fuente: Elaboración propia.

La selección y capacitación del personal permitió durante este período que la ejecución de la contratación del servicio de verificación y calibración por parte de las unidades tanto de atención primaria como secundaria esté al $100 \%$ en ambos casos.

A partir de premisas establecidas se diseñó e implementó la estructura y las funciones para la metrología. En el nivel provincial, dentro de la Dirección de Ciencia e Innovación Tecnológica,

La capacitación de los mismos se realizó a partir del curso formación de capacidades para la metrología dirigidos a la preparación de especialistas C en metrología (Mejías, et al, 2015).

Según Mejías; et al (2015) la constante superación de los recursos humanos, la utilización de los resultados de la ciencia en función de la metrología, el desarrollo de inversiones integrales, el control y las alianzas estratégicas, son elementos claves para garantizar las actividades de aseguramiento metrológico del Sistema Nacional de Salud y contribuir, desde esa óptica, al desarrollo de la salud pública, al mejoramiento de calidad de los servicios y a la satisfacción de la población.

La capacitación de los metrólogos se realizó en función de las plazas a ocupar. A partir de 2014, se observa una disminución en el número de capacitados debido a la estabilidad del personal en las unidades municipales y hospitalarias.

García; et al, (2017) plantea que las instituciones de educación superior tienen la responsabilidad de formar profesionales de acuerdo a las necesidades que presente el mercado, además de que se deben realizar convenios donde se unan el área académica y el área industrial tanto público como privado, para que haya una respuesta acelerada a las necesidades que se presentan en el sector de la salud.

La actividad desarrollada en salud nos acerca al criterio planteado por Hernández Leonard, et al (2018) en cuanto a que los resultados del trabajo realizado en la formación de competencias para la organización y ejecución de los programas de aseguramiento metrológico tienen un impacto tecnológico, por cuanto se han utilizado de manera creativa las herramientas de la investigación-acción en el entrenamiento de los especialistas para la toma de decisiones en normalización, metrología y calidad para las organizaciones. 


\section{Conclusiones.}

- El desarrollo de la formación y capacitación de la actividad metrológica evidencia un uso correcto de los instrumentos de medición, eficiencia en el control metrológico de los equipos y dispositivos médicos que son usados en los servicios de salud y una mejor preparación de los profesionales y técnicos vinculados a la metrología.

- Este trabajo destaca la importancia que tiene la metrología en los equipos biomédicos, y como este impacta en las actividades orientadas a la confiablidad de los resultados, por lo tanto, la formación del personal permitirá a las entidades prestadoras del servicio de salud implementar estrategias de aseguramiento basadas en las normativas internacionales y el cumplimento del decreto ley 183 de 1998 de la metrología.

- A partir de estándares de calidad como son los de la norma ISO 9001: 2015 se puede responder a las necesidades y expectativas de los clientes. Siendo pertinente con las necesidades de formación de los profesionales además de responder a las necesidades de las partes interesadas (Fontalvo, De la Hoz,2018).

\section{Referencias bibliográficas.}

Almeida Rivas, C., HolguónSornoza, E. (2010). Diseño e implantación de un sistema de aseguramiento metrológico en una industria gráfica. (Tesis de Grado), Escuela Superior Politécnica del Litoral Guayaquil, Ecuador. Recuperado de: http://www.dspace.espol.edu.ec/xmlui/handle/123456789/31421

DOI: http://dx.doi.org/10.4067/S0718-50062018000100035

Fontalvo, T. J., De La Hoz, E. J. (2018). Diseño e Implementación de un Sistema de Gestión de la Calidad ISO 9001:2015 en una Universidad Colombiana. Formación Universitaria, 11, 35-44. Recuperado de: http://dx.doi.org/10.4067/S071850062018000100035 .

García, W. U., Orozco Murillo, W., Narvaez, J. G, Palacio, J. A. (2017). Gerencia de mantenimiento, metrología y producción más limpia en equipos y dispositivos médicos en instituciones de salud de Medellín, Colombia. Revista ESPACIOS, 38(52), $28 . \quad$ Recuperado de: www.revistaespacios.com/a17v38n52/a17v38n52p28.pdf

Hernández Leonard, A. R, Filgueiras Sainz de Rozas, M. L, Rodríguez Cardona, J. G. (2018). Generalización de la formación de competencias para organizar y ejecutar el aseguramiento metrológico. Revista de Ingeniería Energética, 39(3), 168-175. Recuperado de:http://scielo.sld.cu/scielo.php?script=sci_arttext\&pid=S181559012018000300004 
Hernández Vargas, L. K., Mesa Rojas, L. M. (2019). Diseño de un Sistema de Gestión de la calidad basado en los requisitos de la norma ISO 9001.2015 en el centro médico por salud. (Ingeniería Industrial), Universidad Autónoma de occidente, Santiago de Cali. http://revistas.ucr.ac.cr/index.php/ingenieria http://scielo.sld.cu.

http://www.cem.es/sites/default/files/la_metrologia_y_su_ensenanza_en_la_universidad.pd f.

LLaque-Lopez, M. (2018). La Infraestructura de la Calidad como Elemento de Desarrollo en el sector saneamiento (Licenciatura en Ingeniería Industrial y de Sistemas). Universidad de Piura, Lima, Perú. Recuperado de: https://hdl.handle.net/11042/3395

Mejías Sánchez, Y., González Labrada, E., Fernández VidalI, A., Hernández Reyes, J., Toledo Fernández, A. M.,Portuondo Sao, M. (2017). Un acercamiento a la Metrología, a través de diez preguntas y respuestas. INFODIR, 26, 83-89. Recuperado de: www.medigraphic.com/pdfs/infodir/ifd-2018/ifd1826k.pdf.

Mejías Sánchez, Y., Morales Suárez, I, PereraVandrell, S. (2015). Bases legales y proyecciones de desarrollo de la metrología en el Sistema Nacional de Salud. Revista Cubana de Salud Pública., 41(1), 172-177. Recuperado de: https://www.scielosp.org/article/ssm/content/raw/?resource_ssm_path=/media/...

Ministerio de Industria, Energía y Turismo. (2016). Formación y difusión de la metrología. Centro Español deMetrología. Recuperado de: https://www.cem.es/sites/default/files/la_metrologia_y_su_ensenanza_en_la_univer sidad.pdf

Oficina Nacional de Normalización. (2015). Norma Internacional NC ISO 9001. Sistemas de gestión de la calidad. Requisitos. Cuba. Recuperado de: http://www.inimet.cubaindustria.cu/

Pacheco Segura, R. (2014). Un modelo para asegurar las mediciones en el sistema de salud pública de Costa Rica. Revista Ingeniería, 24(1), 69-80. Recuperado de:https://revistas.ucr.ac.cr/index.php/ingenieria/article/view/10251/15648

Ramírez Barrera, A. F., Martínez G, J. F, Hidalgo Vásquez, E. (2017). Modelo de gestión para la aplicación del control metrológico legal y la evaluación de la conformidad en equipos biomédicos. Revista Ingeniería Biomédica, 11(21), 65-72. Recuperado de: https://revistas.eia.edu.co/index.php/BME/article/download/1175/1084 
Reyes Ponce, Y., Álvarez Vasallo, L, Hernández Leonard, A. (2011). Importancia de la metrología y su repercusión en el desarrollo. Revista Anales de la Academia de Ciencia de Cuba., 1(1). Recuperado de:www.revistaccuba.cu

Saldarriga Martínez, J. E. (2018). Factores asociados con las competencias metrológicas de un país o región, en el ámbito de su desarrollo, la competitividad y la calidad de vida de las personas (Maestría En Ciencias Naturales y Matemática), Universidad Pontificia Bolivariana Medellín, Colombia. Recuperado de: https://repository.upb.edu.co/bitstream/handle/20.500.11912/4098/FACTORES\%20 ASOCIADOS\%20CON\%20LAS\%20COMPETENCIAS\%20METROL\%C3\%93GI CAS\%20DE\%20UN\%20PA\%C3\%8DS\%200\%20REGI\%C3\%93N.pdf?sequence= $1 \&$ isAllowed=y

Sanchez, G. (2018). Planificación e implementación del análisis de los sistemas de medición en la empresa Denso Manufacturing, bajo los lineamientos del Manual MSA. Universidad Nacional de Córdoba. Recuperado de: http://hdl.handle.net/11086/6255

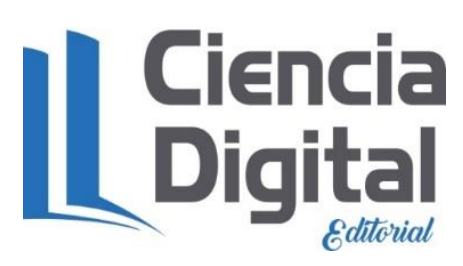




\section{PARA CITAR EL ARTÍCULO INDEXADO.}

Ramos Castro, G., Cárdenas Garabito, D., \& Ávila Segovia, A. (2020). Situación actual y perspectiva de la metrología en el sector salud de la provincia Matanzas. Anatomía Digital, 3(2), 6-15. https://doi.org/10.33262/anatomiadigital.v3i2.1185

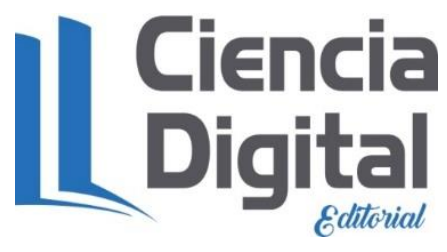

El artículo que se publica es de exclusiva responsabilidad de los autores y no necesariamente reflejan el pensamiento de la Revista Anatomía Digital.

El artículo queda en propiedad de la revista y, por tanto, su publicación parcial y/o total en otro medio tiene que ser autorizado por el director de la Revista Anatomía Digital.
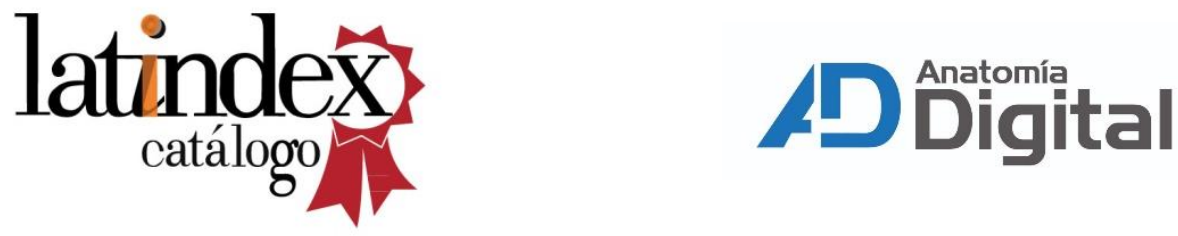